\title{
Reliable multiplex sequencing with rare index mis-assignment on DNB-based NGS platform
}

\author{
Qiaoling $\mathrm{Li}^{1,2,4+}$, Xia Zhao ${ }^{1,2,4+}$, Wenwei Zhang ${ }^{1,2,7 \dagger}$, Lin Wang ${ }^{3}$, Jingjing Wang ${ }^{1,2}$, Dongyang Xu ${ }^{1,2}$, Zhiying Mei ${ }^{4}$, \\ Qiang Liư ${ }^{5}$, Shiyi Du ${ }^{4}$, Zhanqing Li ${ }^{1,2,4}$, Xinming Liang ${ }^{4}$, Xiaman Wang ${ }^{5}$, Hanmin Wei ${ }^{4}$, Pengjuan Liu ${ }^{1,2,4}$, Jing Zou ${ }^{4}$, \\ Hanjie Shen 1,2,4 Ao Chen ${ }^{1,2}$, Snezana Drmanac ${ }^{1,3}$, Jia Sophie Liu ${ }^{3}$, Li Li ${ }^{1,2}$, Hui Jiang ${ }^{4}$, Yongwei Zhang ${ }^{1,3}$, \\ Jian Wang ${ }^{1,6}$, Huanming Yang ${ }^{1,6}$, Xun $\mathrm{Xu}^{1,2}$, Radoje Drmanac ${ }^{1,2,34^{*}}$ and Yuan Jiang ${ }^{3^{*}}$ (D)
}

\begin{abstract}
Background: Massively-parallel-sequencing, coupled with sample multiplexing, has made genetic tests broadly affordable. However, intractable index mis-assignments (commonly exceeds 1\%) were repeatedly reported on some widely used sequencing platforms.

Results: Here, we investigated this quality issue on BGI sequencers using three library preparation methods: whole genome sequencing (WGS) with PCR, PCR-free WGS, and two-step targeted PCR. BGI's sequencers utilize a unique DNA nanoball (DNB) technology which uses rolling circle replication for DNA-nanoball preparation; this linear amplification is PCR free and can avoid error accumulation. We demonstrated that single index mis-assignment from free indexed oligos occurs at a rate of one in 36 million reads, suggesting virtually no index hopping during DNB creation and arraying. Furthermore, the DNB-based NGS libraries have achieved an unprecedentedly low sample-to-sample mis-assignment rate of 0.0001 to $0.0004 \%$ under recommended procedures.
\end{abstract}

Conclusions: Single indexing with DNB technology provides a simple but effective method for sensitive genetic assays with large sample numbers.

Keywords: NGS, Multiplex sequencing, Rare index mis-assignment, DNA nanoball technology

\section{Background}

NGS technology, with its remarkable throughput and rapidly reduced sequencing cost in the current "Big Data" era, is advancing into clinical practice faster than expected by Moore's Law. Updated sequencers, such as Illumina's HiSeq and NovaSeq and BGI's BGISEQ and MGISEQ, are capable of producing hundreds of gigabases to a few terabases of sequencing data in a single run. Different sequencing platforms share a basic NGS workflow, which includes sample/library preparation (nucleic acid isolation, end repair, size selection, adapter

\footnotetext{
* Correspondence: rdrmanac@completegenomics.com; rade@completegenomics.com; yjiang@completegenomics.com

${ }^{\dagger}$ Qiaoling Li, Xia Zhao and Wenwei Zhang contributed equally to this work. 'BGI-Shenzhen, Shenzhen 518083, China

${ }^{3}$ Complete Genomics Inc., 2904 Orchard Pkwy, San Jose, California 95134, USA

Full list of author information is available at the end of the article
}

addition, and optional PCR amplification), sequencing (quality control of the library, DNA cluster/array generation, and instrument operation), and data analysis (quality control, data pipeline analysis, and data interpretation) $[1,2]$. One of the most common strategies for maximizing efficiency is the multiplexing of samples; a unique index is appended to each sample, and multiple samples are pooled together for sequencing in the same run. After sequencing the library pool including the indexes, each read would then be reassigned to its corresponding sample according to the unique index sequence. This sample multiplexing occurs during library preparation, and indexes can be embedded in DNA constructs in two distinct ways-through ligation using indexed adapters or through PCR amplification using indexed primers.

(C) The Author(s). 2019 Open Access This article is distributed under the terms of the Creative Commons Attribution 4.0 International License (http://creativecommons.org/licenses/by/4.0/), which permits unrestricted use, distribution, and reproduction in any medium, provided you give appropriate credit to the original author(s) and the source, provide a link to the Creative Commons license, and indicate if changes were made. The Creative Commons Public Domain Dedication waiver (http://creativecommons.org/publicdomain/zero/1.0/) applies to the data made available in this article, unless otherwise stated. 
However, researchers must be very careful when analyzing de-multiplexed data because index mis-assignment from multiplexing affects data quality and may lead to false conclusions. Index switching can be introduced during many stages of the library preparation and sequencing and post-sequencing processes, including oligo manufacture error or contamination, reagent contamination during experimental handling, template switching during PCR amplification (recombinant PCR), sequencing artifacts or errors, and bioinformatic errors. For example, Illumina's platforms, especially the ones using the new Illumina clustering chemistry, ExAmp, were reported by different labs to have a total index mis-assignment rate of 0.25 to $7 \%$ using dual-indexed adapters [3-5]. Although the results would be unaffected or only minimally affected for users who follow the best practices suggested from Illumina's white paper, sequencing to detect low-frequency alleles such as in liquid biopsy or tumor exome sequencing [6], or single cell sequencing [4] could be seriously impacted with single or regular combinatorial dual indexing $[3,5]$.

Here, we demonstrate that using the PCR-free DNA array preparation and sequencing technology of DNB nanoarrays with optimized library preparation protocols and index quality filters, BGI sequencers even with single indexing are practically free from index switching. We observed nearly zero index hopping from free indexes and an individual sample-to-sample leakage rate in each sequencing lane less than $0.0004 \%$. The total index contamination rate was also orders of magnitude lower than the reported index hopping rate on Illumina's sequencers.

\section{Results}

High indexing fidelity expected for DNA nanoball technology

BGISEQ platforms load DNBs onto patterned arrays and utilize combinatorial Probe Anchor Synthesis (cPAS) for sequencing [7]. The unique DNB technology employs Phi29 polymerase, which has strong strand displacement activity, and the rolling circle replication (RCR) process to enable linear amplification; each amplification cycle remains independent by using the original circular (single-stranded circle) template (Fig. 1a). Therefore, even if errors such as index hopping from incorrectly indexed oligos occur, the false copies will not accumulate. Correct sequences would always be replicated in later DNA copies to ensure the highest amplification fidelity. Thus, we hypothesize that the index hopping should be efficiently prevented on BGI sequencers. To test this hypothesis, we first analyzed two important controls.

\section{Index mis-assignment in controls}

The standard WGS library construction method for BGISEQ-500 includes the following major steps: 1) DNA fragmentation, 2) end repair and A-tailing, 3) indexed adapter ligation, 4) PCR amplification, 5) single-stranded circle (ssCir) formation, and 6) DNB preparation (Fig. 2a). We introduce unique single indexes into every sample during adapter ligation. Each sample is handled separately until samples are pooled, which is known as multiplexing.

To determine whether BGISEQ-500 sequencing accuracy is affected by index hopping, as occurs with Illumina's sequencers $[3,4,8-11]$, we examined the rate of index mis-assignment in BGISEQ-500 runs. We ligated

\section{a Index hopping using DNA nanoball}
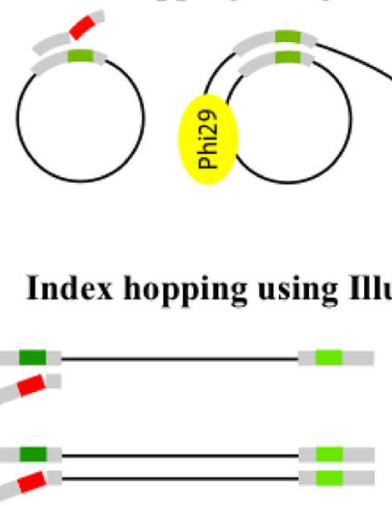

$\underset{\text { after index hopping }}{\stackrel{\text { RCR linear amplification }}{\longrightarrow}}$
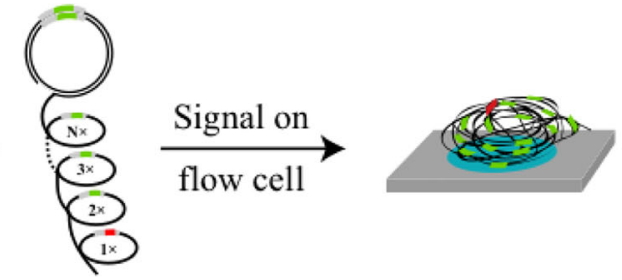

\section{b Index hopping using Illumina's ExAmp chemistry}
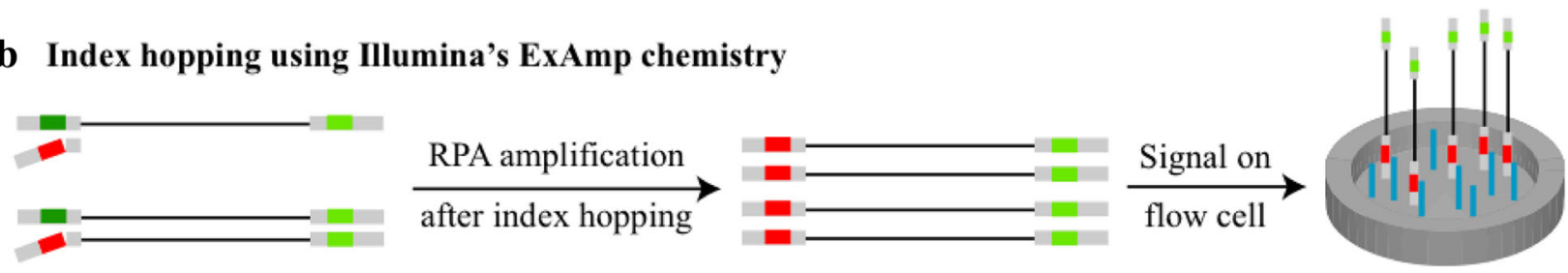

Fig. 1 Mechanisms of index hopping on different sequencing platforms. a Sequencing using DNA nanoball technology is accomplished through Phi29 and RCR linear amplification; each copy is amplified independently using the same template ssCir. In this case, error reads from index hopping cannot accumulate, and most of the signal originates from correct indexes. $\mathbf{b}$ Bridge PCR or ExAmp chemistry utilizes exponential amplification, and index hopping can accumulate as amplification proceeds through each cycle, resulting in mis-assigned samples. Green, correct index; red, wrong index 


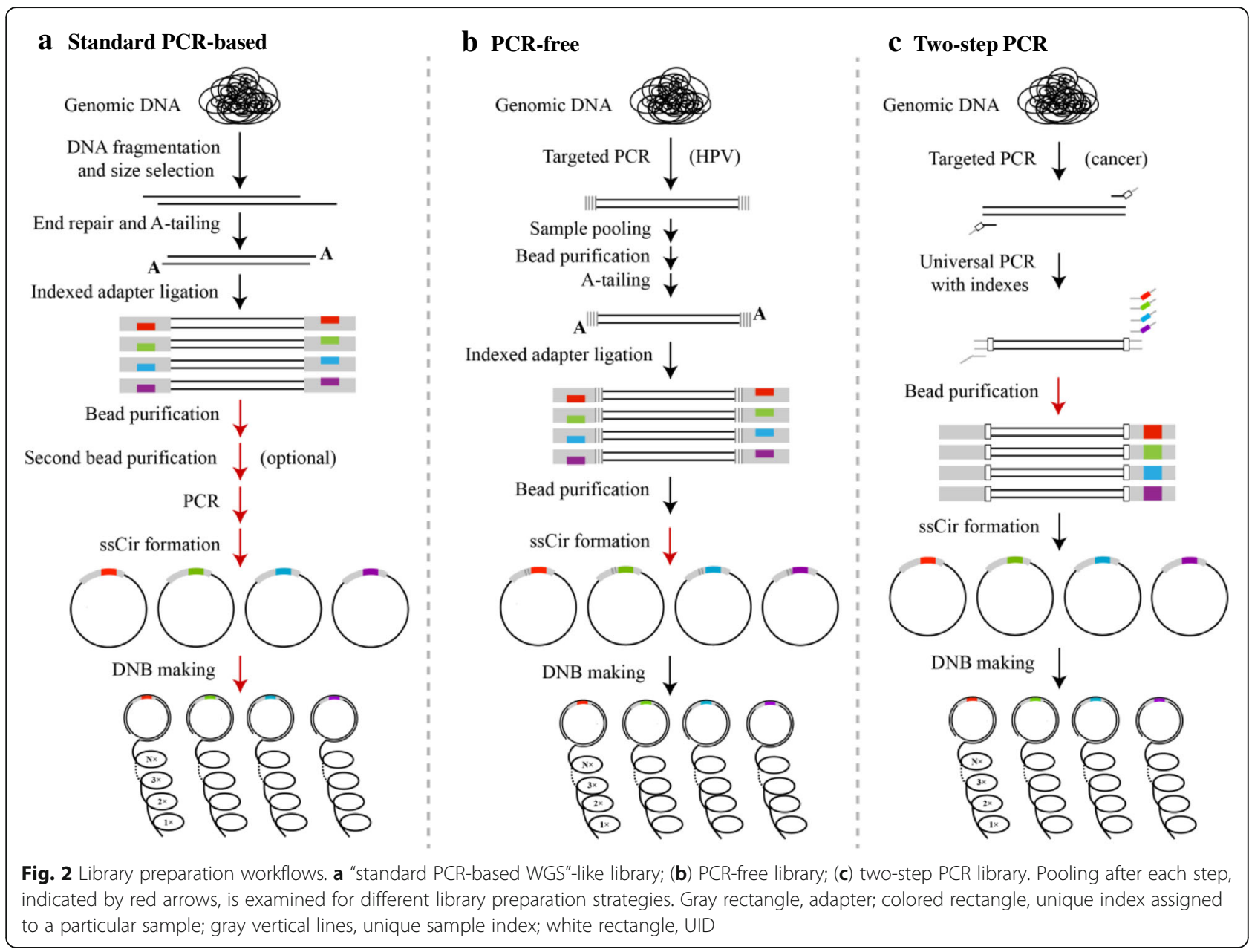

eight unique single indexes to eight gene regions, respectively (indexes 1-8) (Additional file 1: Table S1) or to eight water controls lacking DNA inputs (indexes 3340), and we pooled equal volumes of all samples after PCR amplification. For base positional balance on sequencers, balancing WGS library controls with indexes 41-48 were added at an equal molar ratio prior to DNB preparation (see Methods). To avoid index mis-assignments from oligo synthesis contamination, we ordered indexes 1-8 from IDT (U.S.) and indexes 33-48 from Invitrogen (China) using their regular synthesis services.
The results of assessing different index mis-assignments on BGISEQ-500 are shown in Table 1. All reads passing a quality filter $(\mathrm{Q} 30>60 \%)$ were de-multiplexed with perfect matches on the index regions before mapping to the eight gene regions. Indexes 33-40 were used in empty controls lacking sample DNA. The physical index hopping of the free indexed oligos for all eight indexes occurred at a rate of 2.16E-07 ( 9 out of 41,686,994), 3.11E-07 (14 out of $44,975,628$ ), and $1.40 \mathrm{E}-07$ ( 6 out of $42,875,718$ ) in three repeats (Table 1). In other words, the average per-index probability of this type of index mis-assignment using the

Table 1 Observed frequencies of read mis-assignment in controls

\begin{tabular}{|c|c|c|c|c|c|c|}
\hline \multirow[t]{2}{*}{ Experiments } & \multirow[t]{2}{*}{ Mis-assignment causes } & \multirow[t]{2}{*}{ Index \# } & \multicolumn{3}{|c|}{ Total reads mapped to 8 gene regions } & \multirow{2}{*}{$\begin{array}{l}\text { Mis-assignment } \\
\text { rate per index }\end{array}$} \\
\hline & & & Repeat 1 & Repeat 2 & Repeat 3 & \\
\hline Experimental groups & N.A. & Barcode 1-8 & $41,686,373$ & $44,974,964$ & $42,874,988$ & N.A. \\
\hline Empty controls & Physical barcode hopping & Barcode $33-40$ & 9 & 14 & 6 & 1 in 36 million reads \\
\hline Balancing library controls & Total mis-assignments occur after ssCir & Barcode 41-48 & 612 & 650 & 724 & 1 in 0.5 million reads \\
\hline All groups & All above & All indexes above & $41,686,994$ & $44,975,628$ & $42,875,718$ & N.A. \\
\hline
\end{tabular}

Experimental groups, WGS-like libraries prepared separately using indexes 1 to 8 ; empty controls, indexes 33-40 and reagents used but without sample DNA; balancing library controls, samples prepared and indexed with indexes $41-48$ independently and pooled with test samples after ssCir formation; all groups, total reads of all the indexes. Reads were presented after applying a Q30 > 60\% filter 
DNB platform is 1 in 36 million reads. This number does not exclude index contamination in the experimental handling of indexed oligos, confirming no physical index hopping as we hypothesized.

In another control group, balancing libraries of indexes 41-48 were pooled with experimental samples after ssCir formation and prior to the DNB construction process. The average mis-assignment rate from this control group was $1.92 \mathrm{E}-06(<0.0002 \%, 1$ in 500,000$)$ per index (total reads with indexes 41-48 mapped to genes 1-8 divided by the total reads of all indexes and then divided by 8 ). When a $\mathrm{Q} 30>60 \%$ filter was applied to remove more low-quality indexes, we found two mismatched reads per million mapped reads per index (Additional file 1: Table S2). These rare index mis-assignments from balancing library controls represent all mis-assignments that occurred after the single-stranded circles formation step, which includes index hopping during DNB creation, sequencing or bioinformatic errors, and other mis-assignments during DNB sequencing.

These controls demonstrated that the BGISEQ platform suffers practically no index hopping from excess free indexed oligos and exceptionally low total mis-assignments from the DNB arraying and sequencing processes. In contrast, Costello M. et al. recently reported index hopping rates of 1.31 and $3.20 \%$ for i7 and i5 adapters respectively between a human and an E.coli library using Illumina's ExAmp chemistry [5]. Furthermore, 689,363 reads resulted from uncorrectable double index switching in a total of $842,853,260$ mapped reads. Therefore, i7 and i5 were both swapped in the same DNA, causing sample-to-sample mis-assignment at a rate of $0.08 \%(689,363 / 842,853,260)$, or 1 mis-assignment in 1223 reads. The switching mainly originates from index hopping during ExAmp reactions as their empirical data suggested and results in part from oligo synthesis, handling contamination, or index misreading.

Higher contamination from balancing library controls (indexes 41-48) compared with empty controls (indexes 33-40) suggests that there are some other mechanisms of mis-assignment in DNB sequencing process independent of the physical hopping of free indexed oligos. We further investigated these mechanisms to optimize our library preparation protocol and minimize sample barcode mis-assignments.

\section{Contamination rate observed in "standard PCR-based WGS"-like library construction method Index mis-assignment rates for "standard PCR-based WGS"- like libraries}

To pinpoint an optimal step for sample pooling, we compared the contamination rates of pooling at different processing steps for indexes 1-8 (Figs. 2a and 3a). Each experimental method was repeated in triplicate; therefore, a total of fifteen multiplexed libraries were loaded and sequenced on fifteen lanes of BGISEQ-500.

The overall sequencing quality among all libraries was consistently good, and the mean Q30 score is $91.80 \%$. Before mapping, we de-multiplexed the reads based on their individual indexes allowing for a 1-bp mismatch. The splitting rates were quite uniform among the eight indexes if pooling occurred after PCR amplification. An example of the index split rate for PCR-pooled libraries is shown in Fig. 3b. We next mapped all reads to the reference genome, and the mapping rates were $99.20 \%$ on average. The read numbers of eight gene regions were counted and Fig. 3c shows an example of the read counts mapped for each index at each gene region. The total index contamination was calculated by dividing the sum of all hopped reads by the total reads of all the indexes.

The total index contamination rates, implying index hopping of the sequencing lane among indexes 1 to 8 , were summarized in Fig. 3a for each pooling scenario; the number dropped significantly from $2.6792 \%$ with one bead purification (Ad-1B group) to $0.1365 \%$ when an additional step of bead purification (Ad-2B group) was included to further remove excess adapter oligos after adapter ligation (Fig. 3a, Additional file 1: Table S4), consistent with expectations observed by others [5]. The effect of template switching on index contamination can be further eliminated by pooling after PCR amplification. Therefore, the rate was reduced by an additional 7 -fold, to $0.0183 \%$ (PCR group in Fig. 3a), if samples were pooled after PCR amplification. Libraries pooled after DNB formation demonstrated a total contamination rate less than $0.015 \%$ (DNB group in Fig. 3a). However, pooling after ssCir or DNB formation would slightly increase labor and cost. Taking all of the above into consideration, we conclude that pooling after PCR amplification is optimal to achieve low index contamination.

\section{Explaining and reducing the observed index mis-assignment} Index contamination can be introduced through experimental handling, PCR errors, sequencing errors, oligo synthesis errors, or arraying/clustering methods. We therefore investigated some of these potential causes of the index mis-assignment using the triplicate libraries pooled after PCR in Fig. 3a. First, each mismatch from index 1 to index 8 was retraced to the corresponding DNB and analyzed for sequencing quality. These mismatched DNBs exhibited slightly lower quality scores (average Q30 $=79.24 \%$ ) at the genomic region compared with those of the DNBs with correctly assigned indexes (average Q30 $=89.11 \%$ ). However, the average Q30 of the index region on mismatched DNBs was only $36.66 \%$, which is significantly lower than that of the index region 
a

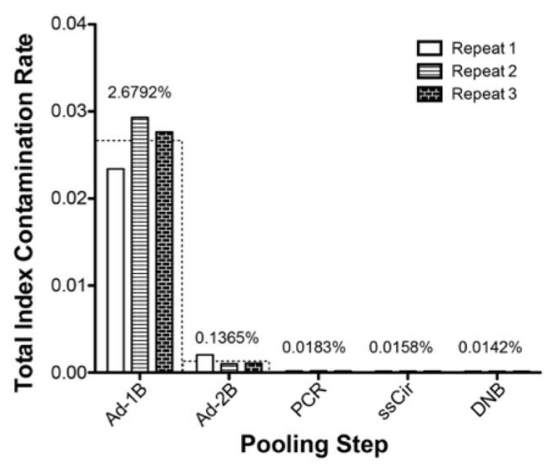

b

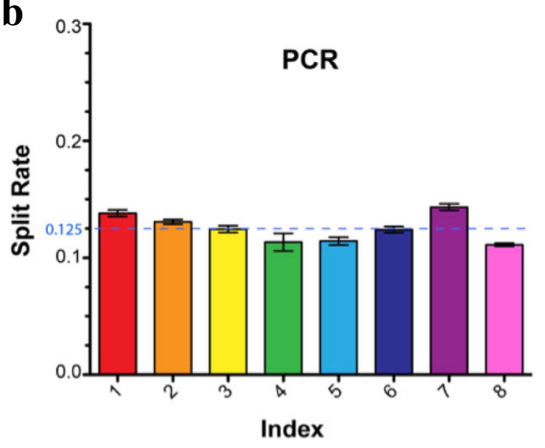

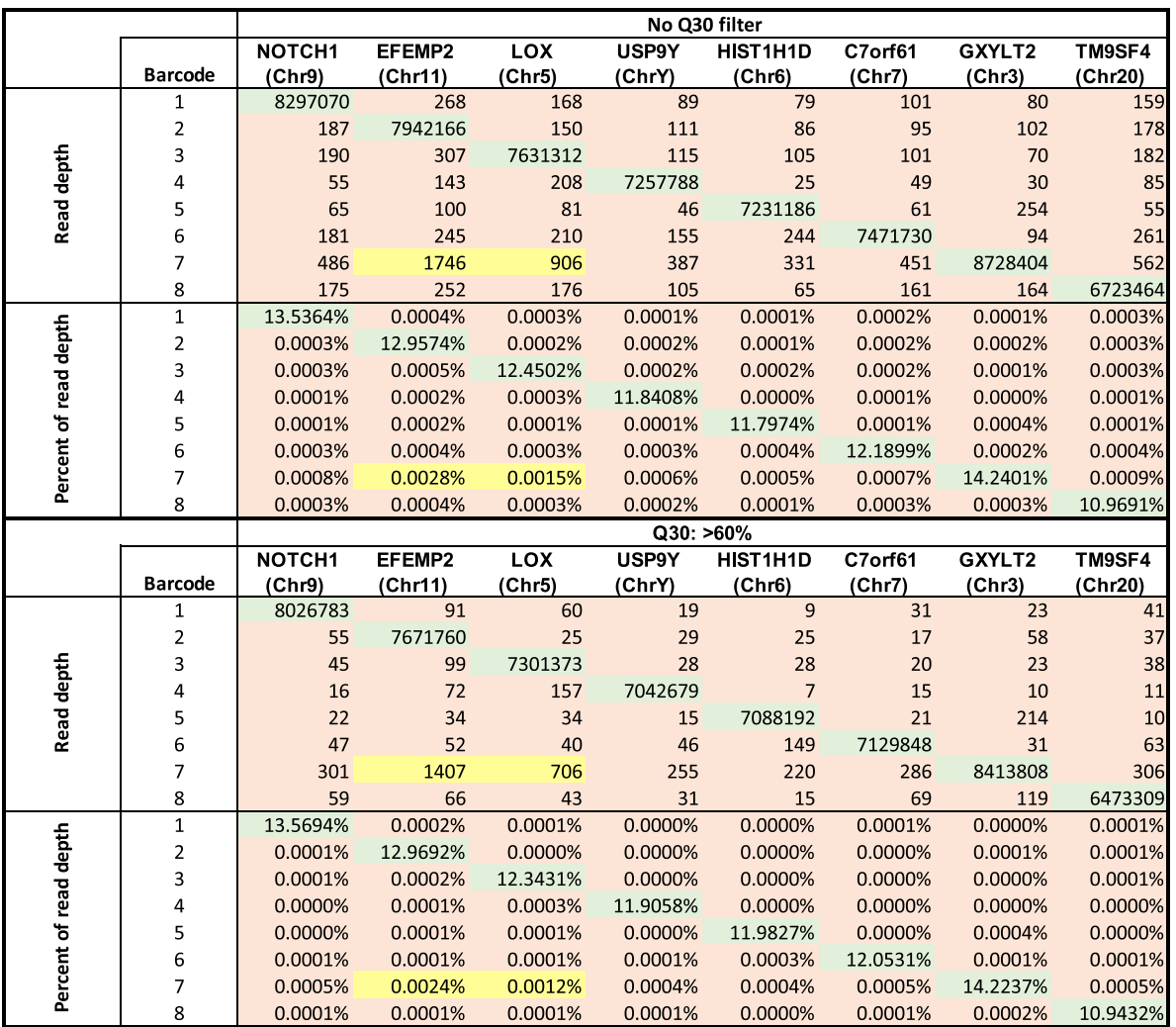

Fig. 3 a Total contamination rates for each pooling scenario. Three replicates are presented with different types of bars. Wider bars with dashed borders represent the average of the three replicates, the exact values of which are labeled on top. The exact values are shown in Additional file 1 : Table S3. $\mathbf{b}$ index split rates when pooling was performed after PCR amplification. Average \pm standard deviation (SD) of three replicates is presented. The theoretical split rate for each index is 0.125. c index contamination matrix when pooling occurred after PCR purification. Indexes 1 to 8 were assigned to Notch1, EFEMP2, Lox, USP9Y, HIST1H1D, C7orf61, GXYLT2, and TM9SF4 respectively. Read numbers and percentages are shown with or without Q30 filter application. Green shading, proper combinations; brown and yellow shading, improper combinations; yellow shading, improper combinations likely resulting from contamination during oligo synthesis. Index contamination rates were calculated by dividing the sum of contaminated reads by the sum of total reads for all eight indexes

for the correctly matched DNBs (average Q30 =91.19\%). These analytical results suggested that in these rare cases in which the true index was not detected, a low-quality false index was assigned. We further questioned whether the mis-assignment in this scenario occurred due to signal bleeding from neighboring DNBs to the affected DNBs. We retraced the positions of DNBs on a chip and calculated the percentage of DNBs that shared the same index sequence with at least one of their four surrounding DNBs. On average, $20.21 \%$ of correctly assigned DNBs shared the same index sequence with their neighboring DNBs; however, this percentage was $57.04 \%$ for mis-assigned DNBs (Additional file 1: Table S5). This result suggested that signal bleeding caused barcode mis-assignment in DNBs that had non-detectable true index signals. Nevertheless, most of these mis-assignments can be adequately removed by 
implementing a Q30 filter; the total contamination rate of indexes $1-8$ dropped from 0.0188 to $0.0097 \%$ and the average sample-to-sample mis-assignment rate dropped to $0.0001 \%$ after applying a Q30>60\% filter for these PCR-pooled libraries (Fig. 3c).

Second, we observed in every run that a higher percentage of reads, especially EFEMP2 and LOX, were mistakenly reassigned to index 7 (highlighted in yellow in Fig. 3c). Through thorough investigation, we found that the majority of these EFEMP2/LOX reads mis-assigned to index 7 were perfectly matched and that the quality was high at the index region (average Q30 = 85.03 and $82.38 \%$, respectively). However, the hamming distance between indexes 2 and 7 is 8 , and the hamming distance between indexes 3 and 7 is 9; therefore, the exceptionally highly contaminated EFEMP2/LOX reads even with the Q30>60\% filter were less likely to be caused by random sequencing errors. The high index mis-assignment rate of barcode 7, which cannot be removed by Q30 > 60\% filter is highly repeatable in different batch of experiments. Different experiments were conducted by different lab technicians in different labs. Indexed oligos in this experiment were ordered using IDT's regular oligo synthesis pipeline instead of TruGrade oligo synthesis, which is specifically advertised for NGS. It is highly likely that the index 7 oligo contaminated all other oligos during synthesis or oligo handling. Because reads of index 7 consisted of both correct and false reads that cannot be differentiated, we excluded data from index 7 , which reduced the total contamination rate by $32 \%$ from $0.0183 \%$ (PCR group in Fig. 3a) to only $0.0124 \%$ (Fig. 4, Additional file 1: Table S6). The rate is further reduced by 64 , to $0.0045 \%$, after applying the Q30 $>60 \%$ filter, whereas the percentage of total reads only dropped by $4 \%$ (Fig. 4, Additional file 1: Table S6). This evidence suggested that oligo synthesis contamination was another major cause of index mis-assignment in this experiment. The average individual index contamination rate is approximately $1-2$ reads/million after removing low-quality reads and oligo contamination (Fig. 3c).

\section{Contamination rate of PCR-free library construction pipeline}

To determine whether our rare contamination rate is sustained when the PCR-free library preparation pipeline is used, we evaluated the SeqHPV protocol with six HPV-positive control samples on the BGISEQ-500. In addition to the aforementioned WGS-like library preparation method, a PCR-free workflow is also commonly used in real-world NGS applications such as PCR-free WGS libraries. Another example is BGI's SeqHPV genotyping assay, which utilizes targeted PCR amplification to first enrich the L1 capsid gene region of human papillomavirus (HPV) and then uses a PCR-free protocol for library preparation (Fig. 2b).

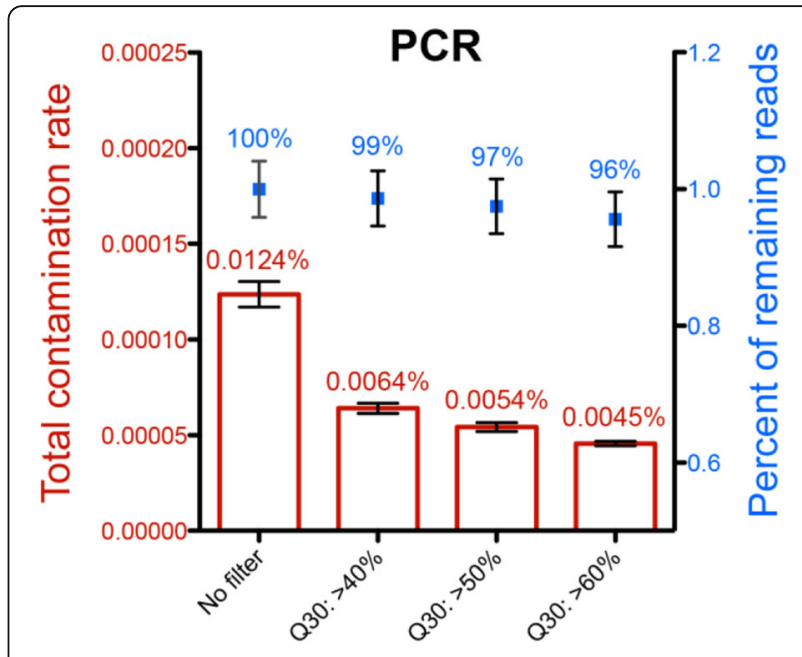

Fig. 4 The effect of filter on total contamination rate and percent of remaining reads. The reads when library pooling occurred after PCR amplification were filtered. Total contamination rate is shown in red and percent of remaining reads is shown in blue. Reads with index 7 were excluded from the calculation. Mapped reads were filtered by different criteria for the Q30 score. Averages \pm SD of three replicates are presented. The average values are labeled on top

The 6 positive samples along with 62 negative samples with $\mathrm{YH}$ genome (an Asian male diploid genome) and 4 water controls were individually amplified with unique sample indexes (Table 2a). Twelve samples from the same row were pooled together after PCR amplification, and then they were ligated with a unique library index (Table 2a, Fig. 2b). Two empty controls without PCR amplicons were included in the ligation; these were separately tagged by index 7 or 8 . The eight libraries were mixed together after ssCir formation and were then subjected to sequencing. After demultiplexing with perfect matches to designed barcodes, BGI's HPV panel precisely detected all six positive samples without any false positive or false negative calls (Table 2b). In our assay, we applied quality controls starting from the targeted PCR step, during which four water controls were used to reveal potential sample contamination during PCR amplification. Reads in the water controls were near zero, suggesting no contamination from targeted PCR (Additional file 1: Table S7). When calculating contamination rates for empty controls, we excluded index 7 because of its oligo synthesis contamination as discussed above. Consistent with our previous findings, the empty control, index 8 , had only $0.0002 \%$ leakage (27 out of 14,582,466) from all of the $H B B$ reads (Table 2c). This 99.9998\% precision without any Q30 filter confirms again that the DNB preparation and arraying strategy can minimize index contamination to a great extent. Similar to the WGS library above, the individual sample-to-sample contamination rate was approximately 4 reads/million on average. The total PCR-free library 


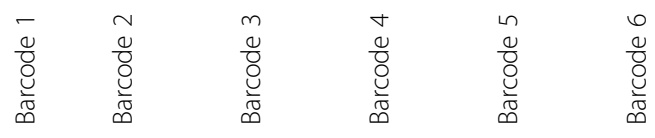

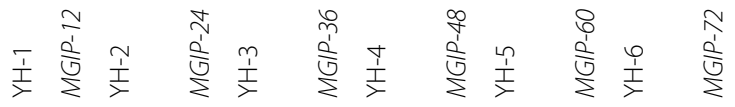

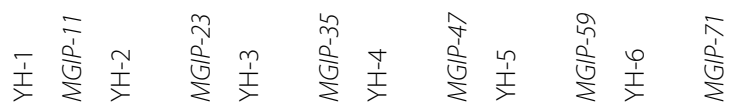

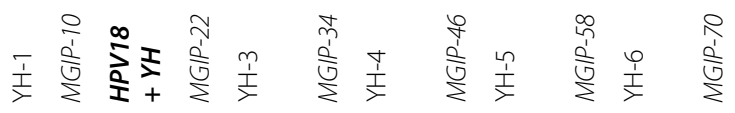

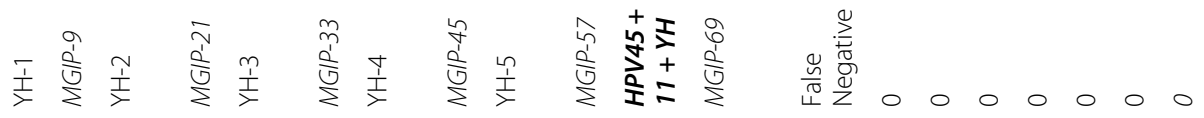

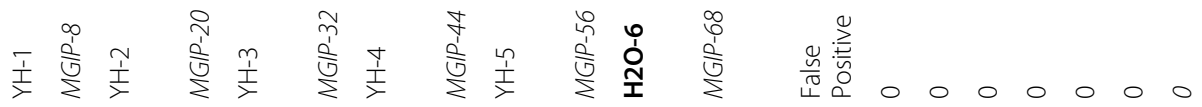

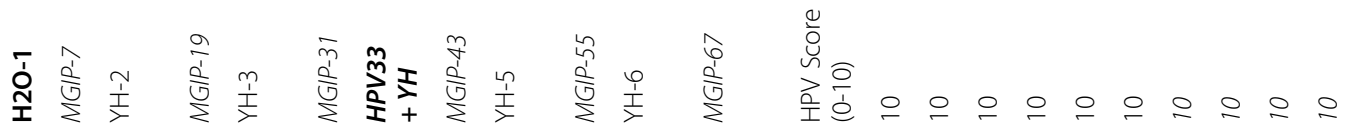

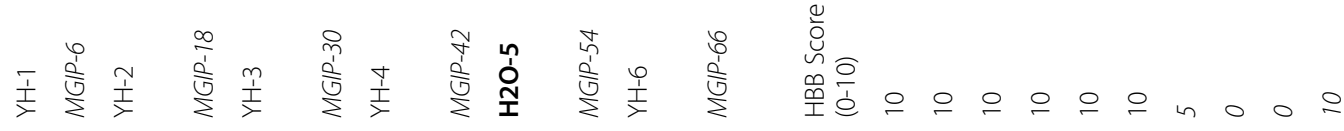

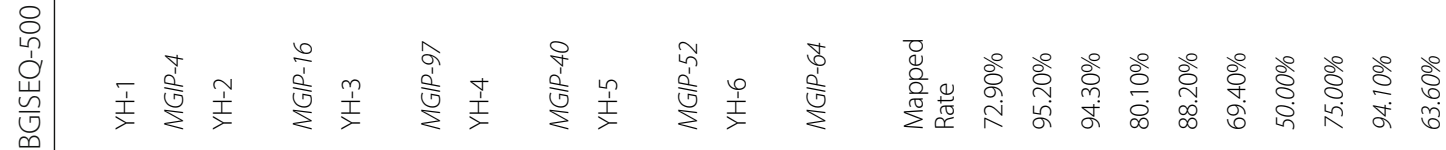

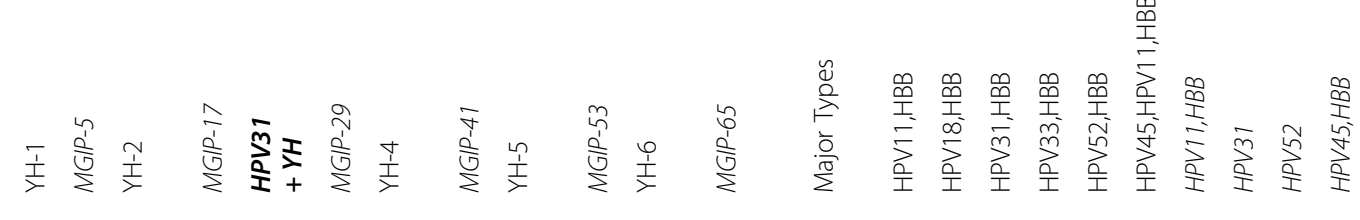

ธ

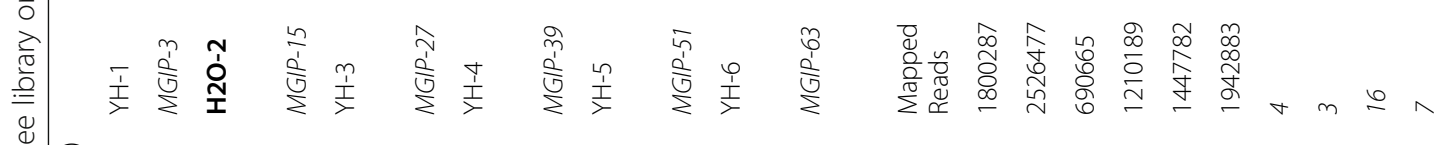

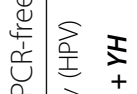

흔

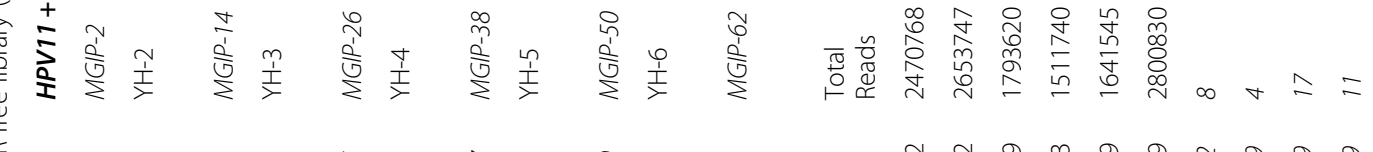

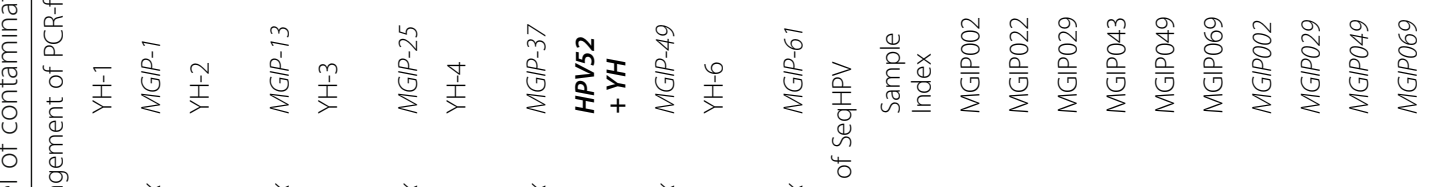

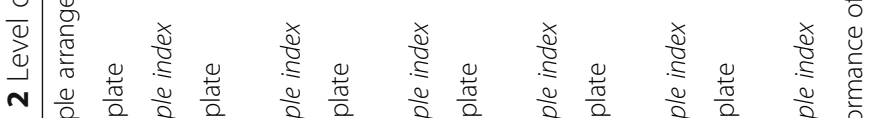

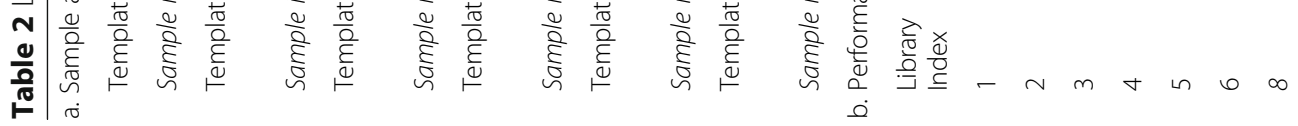




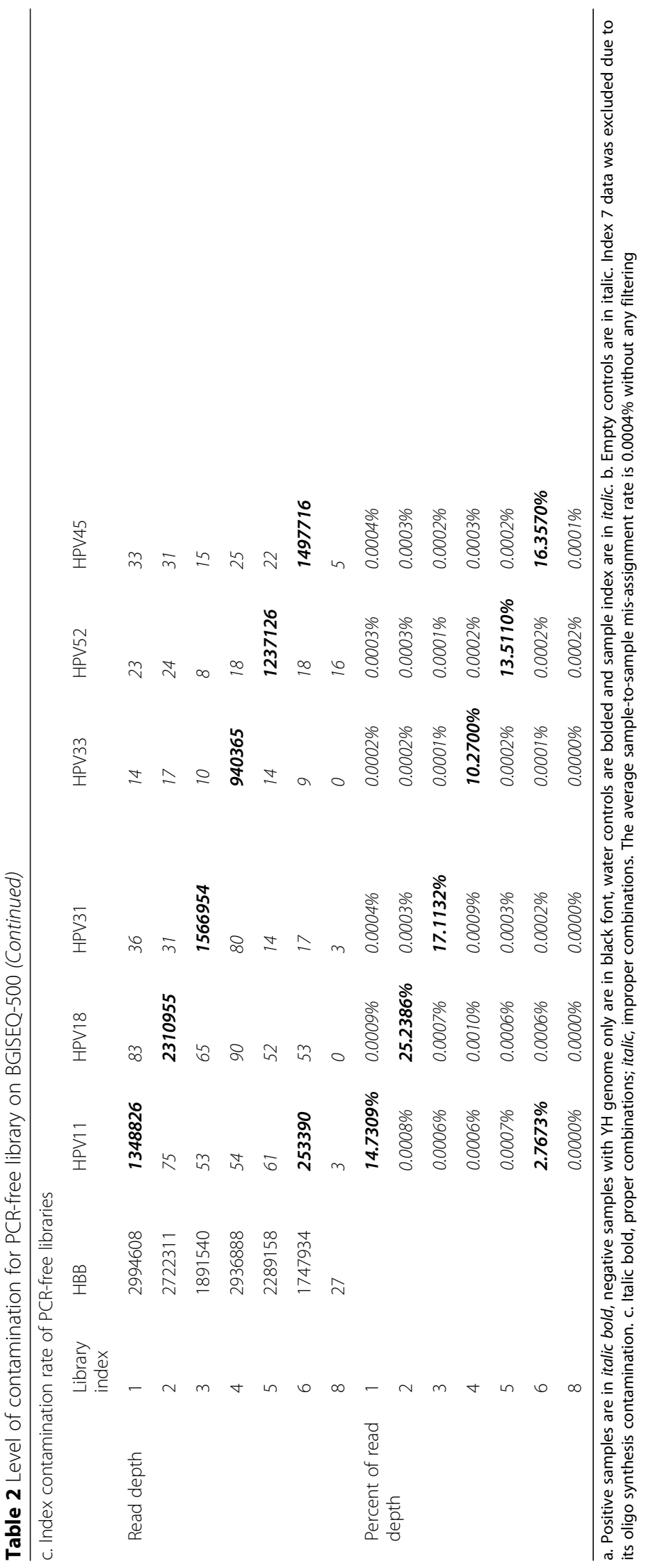


index contamination rate is as low as $0.0118 \%$ without any filtering (Table 2c).

\section{Contamination rate of two-step PCR library preparation approach}

To assess the index mis-match rate when an index is embedded during PCR, we used the BGI lung cancer kit, which leverages this style of indexing (Fig. 2c). The libraries were constructed with index 1 associated with negative control $\mathrm{YH}$ DNA, index 2 associated with an EGFR L858R mutation at 1\%, index 3 associated with a KRAS G12D mutation at $10 \%$, and index 4 associated with an EGFR exon 19 deletion at 50\%. NRAS(p.Q61H) is one of the cancer COSMIC sites included in the kit and is used here as a negative control. We employed unique identifiers (UIDs) to correct and remove PCR and sequencing errors [12,13]. Before the removal of duplications using UIDs, index contamination existed at ratios from 0.000 to $0.05 \%$ (mutant reads divided by the sum of mutant reads and reference reads), but all of these were called "negative" after bioinformatics analysis (Table 3a). Moreover, most of the mis-identified reads dropped to 0 after duplication removal, especially for EGFR mutants (Table 3b). There are multiple copies of KRAS in the genome and the remaining false positives in KRAS are potentially due to primer specificity. A $1 \%$ sensitivity for mutation detection was demonstrated in this study. Taken together, the BGI lung cancer kit verifies that single indexing on DNB sequencing platforms is not susceptible to read mis-assignment and that it can be used for the precise detection of low-frequency somatic variations such as in cancer.

\section{Discussion}

High-throughput sequencing is greatly enhancing the capacity to generate inexpensive and reliable genomic information. Illumina's bridge PCR chemistry is the most widely used clustering mechanism in high-throughput NGS. Illumina recently changed to ExAmp chemistry, which allows cluster generation to occur simultaneously with DNA seeding onto patterned arrays to minimize the likelihood that multiple library fragments are amplified in the same cluster. However, free adapters cannot be completely removed through purification, and with the presence of polymerase and templates, index hopping can be initiated using false adapters [4] (Fig. 1b). Thus, sequencing platforms utilizing ExAmp chemistry are at higher risk of index swapping between samples in a multiplex pool $[3,4,6]$. A recent publication reports dramatically varied index hopping rates with different library construction methods and also indicates that these rates depend on machine types and flow cell batches [5]. PCR-free WGS had the highest total contamination rate of $\sim 6 \%$ [5]. Extra library clean-up, stringent filters, and unique dual indexed adapters have been used to mitigate this problem [11, 14, 15]. Unique dual indexing moves more mis-assigned reads to the "filtered-out reads" compared with regular combinatorial dual indexing. However, the empirical data from Costello M. et al. demonstrated that double index switching could not be filtered out efficiently even with unique dual indexing, and caused 1 error in 1223 reads [5]. Thus, in spite of using unique dual indexes, undetectable index mis-assignment could still occur at rates approaching $0.1 \%$, and could theoretically increase noise in applications that requires high sensitivity, such as low allele frequency or single cell sequencing. Furthermore, this unique dual indexing approach requires complicated and costly adapter and index design, more sequencing directions, and consequently increased sequencing time and cost, and it limits the scalability of multiplexing large numbers of samples.

However, not all sequencing platforms suffer from the index swapping issue. The unique DNB technology used on BGI sequencers for making DNA copies is a linear RCR amplification that is not prone to physical index hopping during DNB preparation and arraying. There are two findings supporting this assertion. First, the empty controls in the control test (index 33-40, Table 1) and in the HPV panel (index 8) have exceptionally low index switching rates from one in 36 million (with filtering) to one in 5 million (without filtering). Second, in the WGS-like library preparation method, balancing libraries with indexes 41-48 were mixed into the pooled libraries (index 1-8). Unlike the mis-assignment of indexes $1-8$, which includes all the contamination starting from library preparation, the mis-assignment of indexes 41-48 only represents the steps after DNB preparation. The average per-index mis-assignment rate for indexes 41-48 (Table 1) is 1 in 500,000 reads to 1 in 1000,000 depending on quality filters, suggesting minimal index mis-assignment during and after DNB preparation and arraying.

We have examined various protocols in detail and found that when pooling is performed after PCR amplification, the index split rates are highly uniform; both index cross-talk in empty controls and total mis-assignment rates are extremely low. Removing apparent oligo synthesis errors can further reduce the total mis-assigned reads by $32 \%$, indicating that oligo quality is most likely the major cause of the remaining index mis-assignment on BGI sequencers. Because single indexing would be affected by oligo quality to a greater extent compared with unique dual indexing, high-quality oligo without any contamination or errors (e.g., nucleotide deletions) is required for the detection of ultralow levels of DNA or diagnostic DNA in DNB-based NGS platforms.

We propose the following practices to maximally avoid index contamination: 1) apply a Q30 filter to increase 


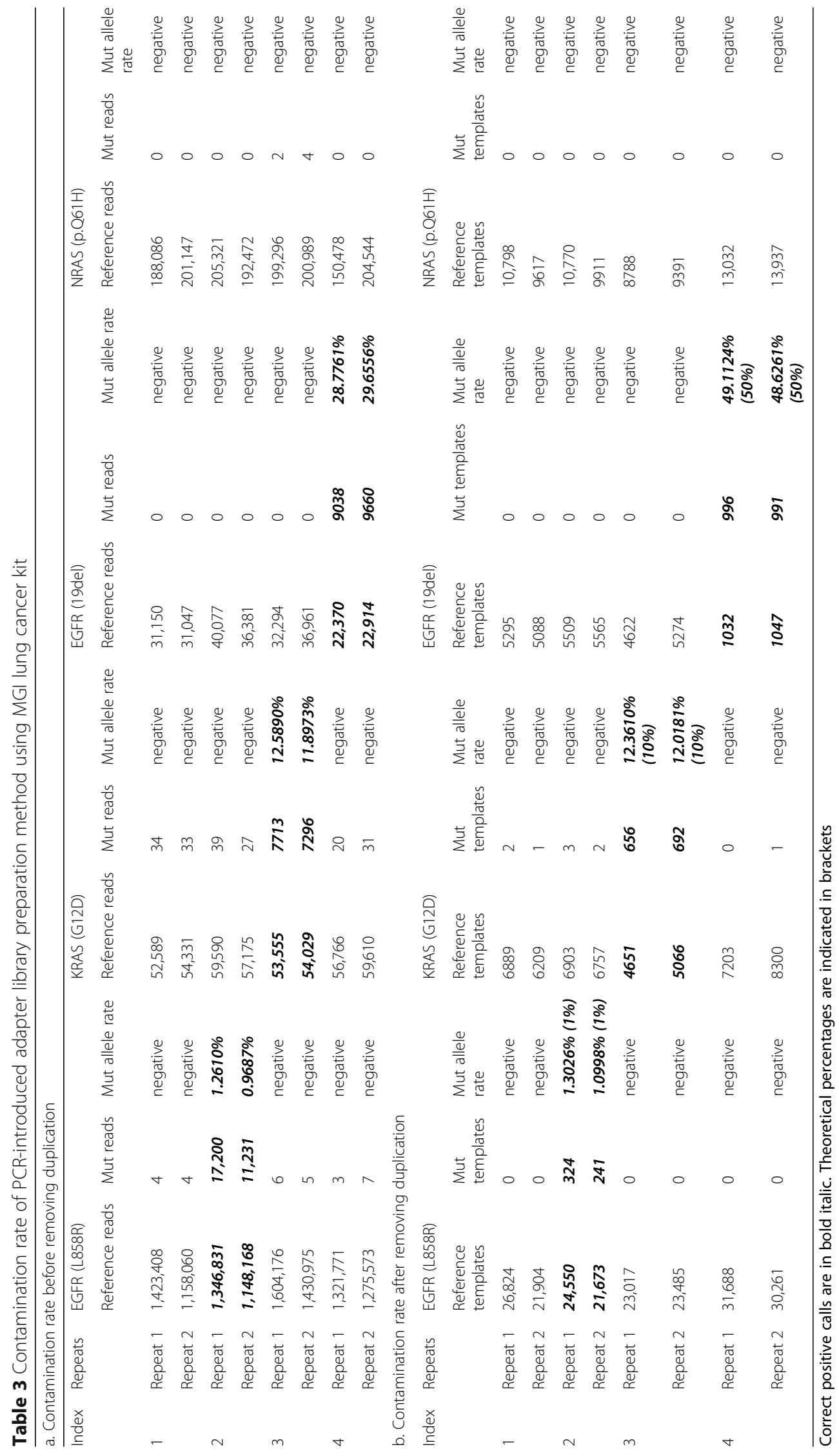


accuracy by removing most sequencing errors, although the quantity of total reads may decrease; 2) pool libraries after PCR amplification; 3) order ultrapure oligos to minimize contamination or artifacts and validate the indexes using an NGS QC method if possible. Using this strategy, the actual individual index mis-assignment rate on the BGI sequencing platform is only $\sim 0.0001-$ $0.0004 \%$ with single indexing; this provides order(s) of magnitude higher precision compared with the unique dual indexing method on newer Illumina platforms (3) and it involves a much simpler adapter structure and fewer sequencing directions.

In summary, the DNB-based NGS platform has rare background-level single index mis-assignment in all frequently used library construction methods we tested, including WGS-like with PCR, PCR-free WGS-like, and two-step targeted PCR libraries, ensuring the best data quality for the NGS community. Single index barcoding is simpler to design, and thus could enable large numbers of samples to be pooled together. Single DNB indexing provides a simple and economical solution for large scale multiplexing, thus aiding more efficient clinical research.

\section{Conclusion}

In this study, we demonstrated that a DNB based NGS platform, achieves exceptionally low index hopping with single indexing. DNB technology utilizes Rolling Circle Replication (RCR) for DNB array preparation. Though only very rare index mis-assignments were observed with the DNB Sequencing technology, we still carefully examined the causes of these mis-assignments in all steps from library preparation to sequencing and data analysis. One source of contamination are oligo synthesis errors/contaminations, resulting in $32 \%$ of the mis-assigned reads. Other root causes include sequencing errors resulting from signal bleeding and other base misreading. We believe that this extreme precision of DNB sequencing technology can help to ease the index hopping concerns for the whole NGS and precision medicine communities. Additionally, single DNB indexing provides a simple and economical solution for large scale multiplexing, thus aiding more efficient clinical research.

\section{Methods}

\section{WGS-like NGS library preparation}

Approximately 400-bp fragments of eight genes (Fig. 2b and Additional file 1: Table S1) were individually amplified by rTaq (Takara Bio, Inc.) and size selected with a $2 \%$ agarose gel (Bio-Rad). Following Agencourt AmpureXP bead purification and quantification with the Qubit ${ }^{\mathrm{Tm}}$ dsDNA HS Assay kit (Thermo Fisher Scientific), single 3 '-A overhangs were added to $100 \mathrm{ng}$ of PCR products through an in-house dA-tailing reaction at $37^{\circ} \mathrm{C}$ for 30 min; heat inactivation was then performed at $65^{\circ} \mathrm{C}$ for $15 \mathrm{~min}$. Adapter ligation was performed at $25^{\circ} \mathrm{C}$ for 30 min in a proprietary ligation mixture containing $1.25 \mu \mathrm{M}$ indexed adapters (regular oligo synthesis through IDT). In the control test, eight empty controls individually tagged with indexes 33 to 40 were incubated with water instead of PCR products for ligation. For Ad-1B- and Ad-2B-pooled libraries, equal masses of the ligated samples with indexes 1 to 8 were mixed after one or two rounds of bead purification, respectively. For all libraries, whether pooled or not, PCR was performed using $1 \mathrm{x}$ KAPA HIFI Hotstart ReadyMix (KAPA) and PCR primers (Invitrogen). After 5 cycles of amplification, $80 \mu \mathrm{L}$ of beads was added to $100 \mu \mathrm{L}$ PCR reactions to clean the reaction. Samples of $20 \mathrm{ng}$ of PCR products with individual indexes were then mixed and used as PCR-pooled libraries. A total of $160 \mathrm{ng}$ of PCR products was used to form single strand circles (ssCir), $10 \mathrm{ng}$ of which was used to prepare DNBs using the SOPs for BGISEQ-500(4). We also pooled indexed samples at equal quantities after ssCir formation (ssCir-pooled libraries) and after DNB preparation (DNB-pooled libraries) based on Qubit ${ }^{\text {Tux }}$ ssDNA quantification. To balance the positional base compositions for sequencing needs, $10 \mathrm{ng}$ of ssCir from a human WGS library control with indexes 41-48 (Invitrogen, China) was added to the ssCirs of Ad-, PCR- or ssCir-pooled libraries. DNB-pooled libraries were mixed with the balancing library immediately after DNB preparation. This balancing WGS library was constructed as reported previously (4). Each pooling strategy was repeated in triplicate and sequenced for single-end reads of $30 \mathrm{bp}$ and index reads of $10 \mathrm{bp}$ on the BGISEQ-500 platform.

Indexes are designed based on the following rules to ensure the uniqueness: 1 . There is no 3 bases tandem repeat in all sequences; 2 . Reversed and complementation sequence of each index is completely different from any barcode; 3 . Indexes were chosen based on their hanming distance and GC balance after polling.

\section{HPV library preparation}

Control plasmid DNA containing individual HPV genotype $11,18,31,33,45$, or 52 or combinations of these was diluted to 1000 copies per sample and mixed with 5 ng of $\mathrm{YH}$ genomic DNA (Table 2a, Additional file 1: Table S8). These positive control samples were used in three triplicate experiments. YH genomic DNA alone was used as an HPV-negative control, and water was used as a multiplex PCR negative control. Each sample was amplified and tagged individually with a 10-bp MGI sample index during PCR using the BGI SeqHPV panel, which recognizes a broad spectrum of HPV genotypes and $\beta$-globin derived from the $H B B$ gene. Multiplex PCR 
was performed in a 96-well plate (Axygen). Twelve amplified samples were pooled into one, and then bead purification was performed. The amplified DNA was provided with a 3 '-A overhang and ligated to a dT-tailed adapter containing index 1 to 6 independently as described above. Empty controls with water were ligated with adapters containing index 7 or 8 . After ssCir formation, DNA with indexes 1 to 8 was pooled using equal volumes and purified after digestion with exonucleases. The ssCir of the balancing library with indexes 41 to 48 was again added to the ssCirs of pooled experimental samples. The triplicates were sequenced using $100 \mathrm{bp}+$ 10 bp single-end runs on BGISEQ-500.

\section{Cancer panel library preparation}

Reference standard DNA amplified from three NSCLC cell lines was purchased from horizon diagnostics (Cambridge, UK), including the following: EGFR L858R (cat. ID: HD254), KRAS G12D (cat. ID: HD272), and EGFR $\triangle \mathrm{E} 746-\mathrm{A} 750$ (cat. ID: HD251). The DNA carrying EGFR L858R, KRAS G12D, or EGFR $\triangle$ E746-A750 mutations was spiked into wild-type $\mathrm{YH}$ genomic DNA at ratios of $1,10 \%$, or $50 \%$, respectively. YH genomic DNA alone was included as a negative control. A proprietary two-step PCR protocol was used to enrich 181 COSMIC variant loci covered by MGI's lung cancer panel kit (BGI). During thermal cycling, a sample index and molecular UIDs were introduced to individual targeted regions. The indexed oligos used in this assay were purchased from IDT through the TruGrade service. The purified multiplex PCR products were validated on a qubit fluorometer (Thermo fisher), pooled with equal mass, and used to prepare ssCirs and DNBs using standard procedures. A balancing WGS control library was mixed after ssCir formation. The duplicated libraries were sequenced for paired-end 50-bp reads along with a 10-bp index region. The mapping rate and capture rate are both greater than $98 \%$, and the uniformity is above $90 \%$.

\section{Sample QC and NGS statistics}

Raw data in FASTQ format obtained from BGISEQ-500 were split into separate FASTQ files based on specific indexes with $0 \mathrm{bp}$ (for control test) or $1 \mathrm{bp}$ (for all other WGS tests) of allowed mismatch. After FASTQ files with individual indexes were generated, the third BWA algorithm, bwa aln, was then used to align the reads to the human reference genome $h g 38$. BAM files from bwa alignment were analyzed to calculate the contamination rates. The reads with proper combinations of index and amplicon were counted and highlighted in green in Fig. 3c. The reads mismatched to incorrect genomic regions were collected for further error type analysis. The base score Q30 (Sanger Phred+ 33 quality score) was used to assess the sequencing quality at both genomic and index regions. By applying different Q30 filters to the index sequences, we managed to reduce the number of reads with sequencing errors by at least two-fold, and more than $96 \%$ of total reads remain with high quality (Fig. 2b and Additional file 1: Table S6). Total index contamination equals the sum of all hopped reads (data with brown shading) divided by the total reads of all the indexes shown in the tables.

For HPV tests, the raw data were preprocessed based on information from lanes and adapters. Using perfectly matched index reads, fq.gz raw sequencing reads were then re-assigned to each sample, and at the same time index and primer sequences were removed. The remaining reads from targeted PCR were aligned to the reference sequences of $H B B$ and various HPV types using bwa aln. Matched reads no fewer than the corresponding cut-off were called positive.

In the cancer panel, raw FASTQ reads were analyzed by SOAPnuke (version 1.5.6). After trimming the adapter and removing low-quality reads, unique identifier sequence information was retrieved and added into the sequence ID of the clean FASTQ data by an in-house developed bioinformatic pipeline. We also calculated the mapping rate, capture rate (fraction of target reads in all reads), duplication rate, and uniformity (fraction of the amplicons whose depth exceeds $20 \%$ of the average depth in all amplicons). After removing duplication, a BAM file was generated; variant calling was performed by in-house developed software, and indel calling was performed using Genome Analysis Toolkit (v4.0.3.0, GATK Mutect2).

\section{Additional file}

Additional file 1: Table S1. PCR primer sequences for 8 genes. Table S2. Mis-assignment rate of control group with Q30 filter. Table S3. Total contamination rates for each pooling scenario in Fig. 3a. Table S4. Total reads and rates of all WGS libraries (indexes 1-8). Table S5. Percentage of DNA sharing the same barcode with neighbours. Table S6. Effect of Q30 filter on sequencing reads and rates when library pooling is performed after PCR amplification (indexes 1-8). Table S7. Index contamination in water control with PCR-free library. Table S8. Raw data of PCR-free library contamination, 3 lanes. (XLSX $138 \mathrm{~kb}$ )

\section{Abbreviations}

CPAS: combinatorial Probe Anchor Synthesis; DNB: DNA-nanoball; NGS: Next generation sequencing; QC: Quality control; RCR: Rolling circle replication; SD: Standard deviation; ssCir: Single-stranded circle; UID: Unique identifier; WGS: Whole genome sequencing

\section{Acknowledgments}

We would like to acknowledge the ongoing contributions and support of all Complete Genomics and BGI-Shenzhen employees, in particular the many highly skilled individuals that build the BGl sequencers and work in the libraries, reagents, and sequencing groups and make it possible to generate high-quality whole genome data. 


\section{Funding}

This work was supported in part by Shenzhen Peacock Plan. No.KQTD20150330171505310.

\section{Availability of data and materials}

The data reported in this study are available in the CNGB Nucleotide Sequence Archive.

https://db.cngb.org/cnsa; accession number CNP0000071.

All the other data used here are included within the published article and its Additional files.

\section{Authors' contributions}

Conception and design of study: QL, XZ, WZ, HW, AC, YJ; Acquisition of data: $\mathrm{QL}$, Jingjing W., XW, PL, JZ, HS, HJ; Analysis and/or interpretation of data: $Q \mathrm{~L}$, $X Z, W Z, D X, Z M, Q L, S$. Du, ZL, XL, YJ; Drafting the manuscript: QL, XZ, WZ, LW; YJ Revising the manuscript critically for important intellectual content: LW, S. Drmanac, JL, LL, RD, YJ; Supervision: YZ, HY, Jian W., XX, RD, YJ; Fund acquisition: RD; Approval of the version of the manuscript to be published: QL, XZ, WZ, LW, Jingjing W., DX, ZM, QL, S. Du, ZL, XL, XW, HW, PL, JZ, HS, AC, S. Drmanac, JL, LL, HJ, YZ, Jian W., HY, XX, RD, YJ.

\section{Ethics approval and consent to participate}

The Institutional Review Board on Bioethics and Bio-safety of BGI (BGI-IRB), NO. BGI-R027 has approved this study.

\section{Consent for publication}

Not applicable.

\section{Competing interests}

Employees of BGI and Complete Genomics have stock holdings in BGl.

\section{Publisher's Note}

Springer Nature remains neutral with regard to jurisdictional claims in published maps and institutional affiliations.

\section{Author details}

${ }^{1}$ BGI-Shenzhen, Shenzhen 518083, China. ${ }^{2}$ China National GeneBank, BGI-Shenzhen, Shenzhen 518120, China. ${ }^{3}$ Complete Genomics Inc., 2904 Orchard Pkwy, San Jose, California 95134, USA. ${ }^{4} \mathrm{MGl}$, BGI-Shenzhen, Shenzhen 518083, China. ${ }^{5}$ BGI Genomics, BGI-Shenzhen, Shenzhen 518083, China. ${ }^{6}$ James D. Watson Institute of Genome Sciences, Hangzhou 310058 , China. ${ }^{7}$ Guangdong High-throughput Sequencing Research Center, Shenzhen, China.

Received: 22 October 2018 Accepted: 26 February 2019

Published online: 13 March 2019

\section{References}

1. Goodwin S, McPherson JD, McCombie WR. Coming of age: ten years of next-generation sequencing technologies. Nat Rev Genet. 2016;17(6):333-51.

2. Head SR, Komori HK, LaMere SA, Whisenant T, Van Nieuwerburgh F, Salomon DR, Ordoukhanian P. Library construction for next-generation sequencing: overviews and challenges. Biotechniques. 2014;56(2):61-4, 66, 68, passim.

3. Effects of Index Misassignment on Multiplexing and Downstream Analysis (white paper) [https://www.illumina.com/content/dam/illumina-marketing/ documents/products/whitepapers/index-hopping-white-paper-770-2017004.pdf].

4. Rahul Sinha GS, Gulati GS, Ezran C, Travaglini KJ, Wei E, Chan CKF, Nabhan AN, Su T, Morganti RM, Conley SD, Chaib H, Red-Horse K, Longaker MT, Snyder MP, Krasnow MA, Weissman IL. index switching causes "spreadingof-signal" among multiplexed samples in Illumina HiSeq 4000 DNA sequencing. In: Edited by Medicine SUSo. bioRxiv; 2017.

5. Costello M, Fleharty M, Abreu J, Farjoun Y, Ferriera S, Holmes L, Granger B, Green L, Howd T, Mason T, et al. Characterization and remediation of sample index swaps by non-redundant dual indexing on massively parallel sequencing platforms. BMC Genomics. 2018;19(1):332.

6. Vodak D, Lorenz S, Nakken S, Aasheim LB, Holte H, Bai B, Myklebost O, Meza-Zepeda LA, Hovig E. Sample-index Misassignment impacts tumour exome sequencing. Sci Rep. 2018;8(1):5307.
7. Huang J, Liang X, Xuan Y, Geng C, Li Y, Lu H, Qu S, Mei X, Chen H, Yu T, et al. A reference human genome dataset of the BGISEQ-500 sequencer. Gigascience. 2017;6(5):1-9.

8. Davey JW, Hohenlohe PA, Etter PD, Boone JQ, Catchen JM, Blaxter ML. Genome-wide genetic marker discovery and genotyping using nextgeneration sequencing. Nat Rev Genet. 2011;12(7):499-510.

9. Mamanova L, Coffey AJ, Scott CE, Kozarewa I, Turner EH, Kumar A, Howard E, Shendure J, Turner DJ. Target-enrichment strategies for next-generation sequencing. Nat Methods. 2010;7(2):111-8.

10. Andolfatto P, Davison D, Erezyilmaz D, Hu TT, Mast J, Sunayama-Morita T, Stern DL. Multiplexed shotgun genotyping for rapid and efficient genetic mapping. Genome Res. 2011;21(4):610-7.

11. MacConaill LE, Burns RT, Nag A, Coleman HA, Slevin MK, Giorda K, Light M, Lai K, Jarosz M, McNeill MS, et al. Unique, dual-indexed sequencing adapters with UMIs effectively eliminate index cross-talk and significantly improve sensitivity of massively parallel sequencing. BMC Genomics. 2018;19(1):30.

12. Kinde I, Wu J, Papadopoulos N, Kinzler KW, Vogelstein B. Detection and quantification of rare mutations with massively parallel sequencing. Proc Natl Acad Sci U S A. 2011;108(23):9530-5.

13. Christensen E, Nordentoft I, Vang S, Birkenkamp-Demtroder K, Jensen JB, Agerbaek M, Pedersen JS, Dyrskjot L. Optimized targeted sequencing of cell-free plasma DNA from bladder cancer patients. Sci Rep. 2018:8(1):1917.

14. Wright ES, Vetsigian $\mathrm{KH}$. Quality filtering of Illumina index reads mitigates sample cross-talk. BMC Genomics. 2016;17(1):876.

15. Kircher M, Sawyer S, Meyer M. Double indexing overcomes inaccuracies in multiplex sequencing on the Illumina platform. Nucleic Acids Res. 2012; 40(1):e3.

\section{Ready to submit your research? Choose BMC and benefit from:}

- fast, convenient online submission

- thorough peer review by experienced researchers in your field

- rapid publication on acceptance

- support for research data, including large and complex data types

- gold Open Access which fosters wider collaboration and increased citations

- maximum visibility for your research: over $100 \mathrm{M}$ website views per year

At BMC, research is always in progress.

Learn more biomedcentral.com/submissions 